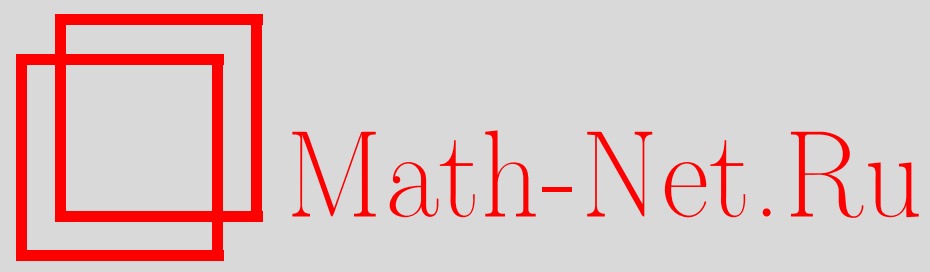

А. А. Махнев (мл.), А. А. Махнев, Овоиды и двудольные подграфы в обобщенных четырехугольниках, Матем. заметки, 2003, том 73, выпуск 6, 878-885

DOI: https://doi.org/10.4213/mzm235

Использование Общероссийского математического портала Math-Net.Ru подразумевает, что вы прочитали и согласны с пользовательским соглашением http://www.mathnet.ru/rus/agreement

Параметры загрузки:

IP: 18.208.226.222

26 апреля 2023 г., 14:43:45

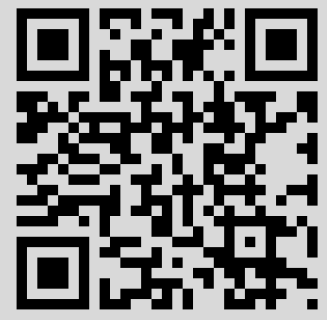




\title{
ОВОИДЫ И ДВУДОЛЬНЫЕ ПОДГРАФЫ В ОБОБЩЕННЫХ ЧЕТЫРЕХУГОЛЬНИКАХ
}

\author{
А. А. Махнев (мл.), А. А. Махнев
}

\begin{abstract}
Система инцидентности, состоящая из точек и прямых, назьвается $\alpha$-частичной геометрией порядка $(s, t)$, если каждая прямая содержит $s+1$ точку, каждая точка лежит на $t+1$ прямой и для любой точки $a$, не лежащей на прямой $L$, найдется точно $\alpha$ прямых, проходящих через $a$ и пересекающих $L$ (обозначение $p G_{\alpha}(s, t)$ ). Если $\alpha=1$, тогеометрия называется обобщенным 4 -угольником и обозначается $G Q(s, t)$. В данной статье установлено, что если псевдогеометрический граф для обобщенного четырехугольника $G Q\left(s, s^{2}-s\right)$ содержит более двух овоидов, то $s=2$. Доказано, что точечный графф обобщенного четырехугольника $G Q(4, t)$ не содержит $K_{4,6}$-подграфов. Наконец, показано, что если в псевдогеометрическом графе для обобщенного четырехугольника $G Q(4, t)$ некоторый $\mu$-подграф̆ содержит треугольник, то $t \leqslant 6$.

Библиография: 5 названий.
\end{abstract}

1. Введение: определения и основные результаты. Мы рассматриваем неориентированные графы без петель и кратных ребер. В данной работе подграфом графа $Г$ будем называть индуцированньй подграф. Если $a, b$ - вершины графа $\Gamma$, то через $d(a, b)$ обозначим расстояние между $a$ и $b$, а через $\Gamma_{i}(a)$ - подграф на множестве всех вершин графа $\Gamma$, которые находятся на расстоянии $i$ от вершины $a$. Подграф $\Gamma_{1}(a)$ назовем окрестностью вершины $a$ и обозначим через $[a]$. Через $a^{\perp}$ обозначим подграф $\{a\} \cup[a]$. Валентностью вершины называется число вершин в ее окрестности. Граф $\Gamma$ назьвается регулярным валентности $k$, если валентность любой вершины $a$ из Г равна $k$. Граб Г назовем реберно регулярным с параметрами $(v, k, \lambda)$, если он содержит $v$ вершин, регулярен валентности $k$, и каждое его ребро лежит в $\lambda$ треугольниках. Граф $\Gamma$ - вполне регулярный граф с параметрами $(v, k, \lambda, \mu)$, если он реберно регулярен с соответствующими параметрами, и $[a] \cap[b]$ содержит $\mu$ вершин для любых двух вершин $a, b$, находящихся на расстоянии 2 в Г. Вполне регулярньй граф называется сильно $p e-$ гулярным графом, если он имеет диаметр 2.

Для подграфа $\Delta$ графа $\Gamma$ через $K_{i}(\Delta)$ обозначим множество вершин из $\Gamma-\Delta$, смежных точно с $i$ вершинами из $\Delta, x_{i}(\Delta)=\left|K_{i}(\Delta)\right|$. Через $K_{m, n}$ обозначим полный двудольньй граф с долями порядков $m, n$. Подграф $[a] \cap[b]$ назовем $(\mu-) \lambda$-nодграфом, если вершины $a, b$ смежны (находятся на расстоянии 2 ).

Система инцидентности, состоящая из точек и прямых, называется $\alpha$-частичной геометрией порядка $(s, t)$, если каждая прямая содержит $s+1$ точку, каждая точка лежит

Работа выполнена при финансовой поддержке Российского фонда фундаментальных исследований, грант № 99-01-00462. 
на $t+1$ прямой (прямые пересекаются не более, чем по одной точке) и для любой точки $a$, не лежащей на прямой $L$, найдется точно $\alpha$ прямых, проходящих через $a$ и пересекающих $L$ (обозначение $p G_{\alpha}(s, t)$ ). Если $\alpha=1$, то геометрия называется обобщенным четырехугольником и обозначается $G Q(s, t)$.

Точечным графом частичной геометрии назьвается граф, вершинами которого являются точки геометрии, и две вершины смежны, если они различны и лежат на общей прямой. Легко понять, что точечньй граф частичной геометрии $p G_{\alpha}(s, t)$ сильно регулярен с параметрами: $v=(s+1)(1+s t / \alpha), k=s(t+1), \lambda=(s-1)+(\alpha-1) t$, $\mu=\alpha(t+1)$. Сильно регулярньй граф, имеющий вьшеуказанные параметры, назьвается псевдогеометрическим графом для $p G_{\alpha}(s, t)$. В таких графах гранища Хоффмана для клик (см. [1]) равна $s+1$, и каждая вершина вне $(s+1)$-клики $L$ смежна с $\alpha$ вершинами из $L$. Овоидом псевдогеометрического графа для $p G_{\alpha}(s, t)$ называется коклика из $s t / \alpha+1$ точек.

В данной статье показано, что если псевдогеометрический граф Г для частичной геометрии $p G_{\alpha}(s, t)$ содержит коклику $\mathscr{O}$, достигающую равенства в границе Хоффмана и гранище Цветковича для коклик (см. [1]), то $\alpha=1, t=s^{2}-s$ и подграф̆ $\Gamma-\mathscr{O}$ сильно регулярен с параметрами $\left(s^{2} t+s,(s-1)(t+1), s-2,(s-1)^{2}\right)$. Далее, установлено, что если псевдогеометрический граф для обобщенного четырехугольника $G Q\left(s, s^{2}-s\right)$ содержит более двух овоидов, то $s=2$. Доказано, что если псевдогеометрический граф для обобщенного четырехугольника $G Q(4, t)$ содержит $K_{4,6}$-подграф, то $t=6$ (в частности, точечньй граф обобщенного четырехугольника $G Q(4, t)$ не содержит $K_{4,6}$-подграфов). Наконец, показано, что если в псевдогеометрическом графе для обобщенного четырехугольника $G Q(4, t)$ некоторьй $\mu$-подграфф содержит треугольник, то $t \leqslant 6$.

ПРЕДЛОЖЕНИЕ 1. Пусть псевдогеометрический граф Г для частичной геометрии $p G_{\alpha}(s, t)$ содержит коклику $\mathscr{O}$, достигающую равенства в граниче Хоффмана и граниче Цветковича для коклик. Тогда $\alpha=1, t=s^{2}-s$ и подграф Грегулярен с параметрами

$$
\left(s^{2} t+s,(s-1)(t+1), s-2,(s-1)^{2}\right)
$$

и собственными значениями

$$
(s-1)\left(s^{2}-s+1\right), \quad s-1, \quad-\left(s^{2}-2 s+2\right)
$$

кратностей

$$
1, \quad(s-1)\left(s^{3}-s^{2}+1\right), \quad s^{3}-s^{2}
$$

соответственно.

Интерес к обобщенным четырехугольникам порядка $\left(s, s^{2}-s\right)$ объясняется еще и тем, что для $G Q(s, t)$ с $1<s \leqslant t$ вьполняется неравенство Хигмена $t \leqslant s^{2}$, причем если $t \neq s^{2}$, то $t \leqslant s^{2}-s$ (см. [2, леммы $\left.\left.1.2 .3,1.2 .5\right]\right)$.

Заметим, что известно существование обобщенного четырехугольника $G Q\left(s, s^{2}-s\right)$ только для $s=2$. В этом случае дополнение овоида является графом Петерсена (сильно регулярньм графом с параметрами $(10,3,0,1)$ ). Далее, $G Q(2,2)$ содержит точно 6 овоидов. Известно, что псевдогеометрический граф для обобщенного четырехугольника $G Q(3,6)$ не существует (см. [3] и независимо [4]). Вопрос о существовании $G Q\left(s, s^{2}-s\right)$ для $s \geqslant 4$ остается открытьм. 
ПРЕДЛОЖЕНИЕ 2. Пусть псевдогеометрический граф Г для обобщенного четырехугольника $G Q\left(s, s^{2}-s\right)$ содержит более двух овоидов. Тогда $s=2$.

Хорошо известно, что если точечньй граф $Г$ для обобщенного четырехугольника $G Q(s, t)$ содержит $K_{m, n}$-подграф, то $(m-1)(n-1) \leqslant s^{2}$. Следующие два результата существенно уточняют эту оценку в случае $s=4$.

ТЕОРемА. Пусть псевдогеометрический граф Г для обобщенного четырехугольника $G Q(4, t)$ содержит $K_{4,6}$-подграф. Тогда $t=6$.

СлЕДСТВИЕ. Точечный граф обобщенного четырехугольника $G Q(4, t)$ не содержит $K_{4,6}-$ подграфов.

В связи с нашей теоремой интерес представляет следующая

ПроБлЕмА. Существует ли псевдогеометрический граф Г для обобщенного четырехугольника $G Q(4,6)$, содержащий $K_{4,6}$-подграф

Для доказательства геометричности псевдогеометрическогографа Г для обобщенного четьрехугольника $G Q(s, t)$ достаточно установить, что $\mu$-подграфы из Г не содержат ребер. Первым приближением к этой цели в случае $s=4$ является удаление треугольников из $\mu$-подграфов.

ПРЕДЛОЖЕНИЕ 3. Пусть в псевдогеометрическом графе Г для обобщенного четырехугольника $G Q(4, t)$ некоторый $\mu$-подграф содержит треугольник. Тогда $t \leqslant 6$.

2. Доказательство основных результатов. Прежде чем перейти к доказательству основных результатов статьи, приведем одну вспомогательную лемму.

Лемма 1. Пусть Г - сильно регулярный граф с параметрами $(v, k, \lambda, \mu), \Delta$ индуцированный подграф с $N$ вершинами, $M$ ребрами и валентностями вершин $d_{1}, \ldots, d_{N}$. Тогда

$$
(v-N)-(k N-2 M)+\left(\lambda M+\mu\left(\left(\begin{array}{c}
N \\
2
\end{array}\right)-M\right)-\sum_{i=1}^{N}\left(\begin{array}{c}
d_{i} \\
2
\end{array}\right)\right)=x_{0}+\sum_{i=3}^{N}\left(\begin{array}{c}
i-1 \\
2
\end{array}\right) x_{i}
$$

əде $x_{i}=x_{i}(\Delta)$.

ДокАЗАТЕЛЬСТво. См. доказательство леммы 1 из [5].

ДоКАЗАТЕЛЬСТВО ПРЕДЛОЖЕНИЯ 1. Пусть Г- псевдогеометрический граф для частичной геометрии $p G_{\alpha}(s, t)$, содержащий коклику $\mathscr{O}$, достигающую равенства в гранище Хоффмана и границе Цветковича для коклик, и $\Sigma=\Gamma-\mathscr{O}$. Тогда

$$
|\mathscr{O}|=1+\frac{s t}{\alpha}=\frac{s(s-\alpha+1)(s t+\alpha)}{\alpha(s+t+1-\alpha)}
$$

поэтому $t=(s-1)(s+1-\alpha)$.

По теореме 4 [1] граф $\Sigma$ сильно регулярен. Покажем, что $\Sigma$ имеет параметры

$$
\begin{gathered}
v=s\left(1+\frac{s t}{\alpha}\right), \quad k=(s-1)(t+1), \\
\lambda=(s-2)(1+(\alpha-1)(s+1-\alpha)), \quad \mu=s^{2}-\alpha s-s+2 \alpha-1 .
\end{gathered}
$$


Далее, если $a, b$ - две смежные (соответственно несмежные) вершины из $\Sigma$, то $[a] \cap[b]$ содержит $1+(\alpha-1)(s+1-\alpha)$ вершин (соответственно $s+(s-1)(\alpha-1)(s+1-\alpha)$ вершин) из $\mathscr{O}$.

Если $K$ является $(s+1)$-кликой из $\Gamma$, то $K$ пересекает овоид $\mathscr{O}$ в единственной точке $x$. Тогда любая точка из $\mathscr{O}-x$ смежна с $\alpha$ вершинами из $K$, поэтому любая пара вершин из $K-\mathscr{O}$ смежна с $1+(\alpha-1)(s+1-\alpha)$ точками из $\mathscr{O}$. Так как граф $\Sigma$ сильно регулярен, то любое ребро из $\Sigma$ смежно с $1+(\alpha-1)(s+1-\alpha)$ точками из $\mathscr{O}$. Отсюда получаются выражения для параметров $v, k, \lambda$. Из прямоугольного соотношения $k(k-\lambda-1)=(v-k-1) \mu$ получим выражение для $\mu$. Наконец, каждая пара несмежных вершин из $\Sigma$ смежна с $\alpha(t+1)-\mu$ точками из $\mathscr{O}$.

Допустим, что $\alpha>1$. Так как каждая вершина вне $\mathscr{O}$ смежна с $t+1$ точками из $\mathscr{O}$, то $s+(\alpha-1) t \leqslant t+1$, поэтому $\alpha=2, s=1$ и $t=0$, противоречие. Отсюда $\alpha=1, \Gamma-$ псевдогеометрический граф для обобщенного четырехугольника $G Q\left(s, s^{2}-s\right)$, граф $\Sigma$ имеет параметры

$$
\left(s^{2} t+s,(s-1)(t+1), s-2,(s-1)^{2}\right),
$$

собственные значения графа $\Sigma$ равны

$$
(s-1)(t+1), \quad s-1, \quad-\left(s^{2}-2 s+2\right)
$$

и имеют кратности

$$
1, \quad(s-1)(s t+1), \quad s t
$$

соответственно. Предложение 1 доказано.

Перейдем к доказательству предложения 2.

ЛЕмМа 2. Если псевдогеометрический граф Г для обобщенного четырехугольника $G Q\left(s, s^{2}-s\right)$ содержит два различных овоида $\mathscr{O}$ и $\mathscr{O}^{\prime}$, то $\left|\mathscr{O} \cap \mathscr{O}^{\prime}\right|=(s-1)^{2}$, причем $\left(\mathscr{O}-\mathscr{O}^{\prime}, \mathscr{O}^{\prime}-\mathscr{O}\right)$ является симметричной $2-\left(v, s^{2}-s+1, s\right)$ схемой, әде $v=s\left(s^{2}-2 s+2\right)$. Далее, любая вершина из $\Gamma-\left(\mathscr{O} \cup \mathscr{O}^{\prime}\right)$ смехна $c s-1$ вершинами uз $\mathscr{O} \cap \mathscr{O}^{\prime}$.

ДокАЗАТЕЛЬСТво. Попредложению 1 для любых двух несмежных вершин вне $\mathscr{O}$ пересечение их окрестностей содержит $s$ точек из $\mathscr{O}$, поэтому $\left(\mathscr{O}-\mathscr{O}^{\prime}, \mathscr{O}^{\prime}-\mathscr{O}\right)$ является симметричной $2-\left(v, s^{2}-s+1, s\right)$-схемой, где $v=|\mathscr{O}|-\left|\mathscr{O}^{\prime} \cap \mathscr{O}\right|$. Но тогда $\left(s^{2}-s\right)\left(s^{2}-s+1\right)=$ $(v-1) s$, поэтому $v=s\left(s^{2}-2 s+2\right)$ и $\left|\mathscr{O} \cap \mathscr{O}^{\prime}\right|=(s-1)^{2}$.

Заметим, что для коклики $\mathscr{O}-\mathscr{O}^{\prime}$ графа $\Gamma-\mathscr{O}^{\prime}$ достигается равенство в границе Хоффмана для коклик, поэтому любая вершина из $\Gamma-\left(\mathscr{O} \cup \mathscr{O}^{\prime}\right)$ смежна с $s-1$ вершинами из $\mathscr{O} \cap \mathscr{O}^{\prime}$. Лемма доказана.

Такие симметричные схемы существуют для $s=2$ и 4 . Пусть $\Lambda$ - граф вершин и ребер обычного куба. Для вершины $a \in \Lambda$ положим $X=\{a\} \cup \Lambda_{2}(a), \mathscr{B}=\Lambda(a) \cup \Lambda_{3}(a)$. Тогда $(X, \mathscr{B})$ дает пример схемы из заключения леммы 2 с $s=2$. Для $s=4$ соответствующим примером является схема проективных точек и гиперплоскостей проективного пространства $P G(3,3)$.

ДоКАЗАТЕЛЬСТВО ПРЕДЛОЖЕНИЯ 2. Пусть $\mathscr{O}, \mathscr{O}^{\prime}$ и $\mathscr{O}^{\prime \prime}$ - три различных овоида из $\Gamma, \Lambda=\mathscr{O}^{\prime \prime} \cap \mathscr{O}^{\prime} \cap \mathscr{O}, \beta=|\Lambda|$. Число ребер между $\left(\mathscr{O} \cap \mathscr{O}^{\prime}\right)-\Lambda$ и $\mathscr{O}^{\prime \prime}-\left(\mathscr{O}^{\prime} \cup \mathscr{O}\right)$ равно $\left((s-1)^{2}-\beta\right)\left(s^{2}-s+1\right)=\left(s^{3}-s^{2}+1-2(s-1)^{2}+\beta\right)(s-1)$. Отсюда $\beta=(s-1) \gamma$ и $(s-1-\gamma)\left(s^{2}-s+1\right)=s^{3}-3 s^{2}+4 s-1+(s-1) \gamma$, следовательно, $\gamma \equiv-1(\bmod (s-1))$ и $\gamma=s-2$. Таким образом, $s^{2}-s+1=s^{3}-3 s^{2}+4 s-1+(s-1)(s-2)$, поэтому $s=2$. Предложение 2 доказано. 
Лемма 3. Точечный граф Г обобщенного четырехугольника $G Q(4, t)$ не содержит $K_{3,9}-u K_{4,7}$-подграфов.

ДокАЗАтЕЛьСтво. Пусть $G Q(s, t)$ содержит $K_{m, n}$-подграф $\Delta$. По лемме 1.4 .1 [2] $(m-1)(n-1) \leqslant s^{2}$, причем в случае равенства либо $m=n=s+1$, либо $s<t, n=1+t$, $m=1+s^{2} / t$. Поэтому в случае $s=4$ граф $Г$ не содержит $K_{3,9}$ и $K_{4,7}$-подграфов.

До конца работы мы будем предполагать, что $\Gamma$ - псевдогеометрический граф для $G Q(4, t)$. По условию целочисленности $t+4$ делит $20 \cdot 12$, поэтому $t=1,2,4,6,8,11,12$ или 16.

ЛЕмма 4. Пусть $\Delta=\left\{a_{1}, \ldots, a_{4} ; b_{1}, \ldots, b_{n}\right\}$ является $K_{4, n}$-подграфом из $\Gamma$, $K_{i}=K_{i}(\Delta), x_{i}=\left|K_{i}\right|$. Тогда выполняются следуюшие утвер ждения:

(1) $x_{0}+\sum\left(\begin{array}{c}i-1 \\ 2\end{array}\right) x_{i}=t(n-4)(n-5) / 2-3(n-1)(n-6) / 2$;

(2) ecлu $n=5$ u $x_{0} \neq 0$, mo $t=4$;

(3) $n<7$.

ДокАЗАТЕЛЬСтво. Число вершин $N$ подграфа $\Delta$ равно $4+n$, а число его ребер $M=4 n$, валентности вершин равны $d_{1}=\cdots=d_{4}=n$ и $d_{i}=4$ для $i>4$. По лемме 1 имеем

$$
x_{0}+\sum\left(\begin{array}{c}
i-1 \\
2
\end{array}\right) x_{i}=\frac{t(n-4)(n-5)}{2}-\frac{3(n-1)(n-6)}{2} .
$$

Если $n=5$ и $w \in K_{0}$, то $x_{0}+\sum\left(\begin{array}{c}i-1 \\ 2\end{array}\right) x_{i}=6$. Число 2-путей с началом $w$ и концом в $\Delta$ равно $9(t+1)$. С другой стороны, число таких 2 -путей не больше $4+3 \cdot 2+2(4 t+1)$, если $[w]$ содержит точку из $K_{4}$, и не больше $3 \cdot 5+2(4 t-1)$, если $[w]$ не пересекает $K_{4}$. Отсюда $9 t+9 \leqslant 8 t+13$ и $t \leqslant 4$. Утверждение $(2)$ доказано.

Допустим, что $n=7$. Положим $\Delta^{\prime \prime}=\Delta-\left\{b_{1}, b_{2}\right\}, M_{i}=K_{i}\left(\Delta^{\prime \prime}\right), y_{i}^{\prime}=\left|M_{i}\right|$. Тогда $y_{0}^{\prime}+\sum\left(\begin{array}{c}i-1 \\ 2\end{array}\right) y_{i}^{\prime}=6$, причем $b_{1}, b_{2} \in M_{4}$. Отсюда $y_{0}^{\prime}=y_{3}^{\prime}=0$ и каждая точка из $\left(\left[b_{1}\right] \cap\left[b_{2}\right]\right)-\Delta$ смежна с некоторой вершиной $b_{j}$ из $\left\{b_{3}, \ldots, b_{7}\right\}$. Теперь $x_{0}(\Omega)+$ $\sum\left(\begin{array}{c}i-1 \\ 2\end{array}\right) x_{i}(\Omega)>6$ для подходящего $K_{4,5}$-подграфа $\Omega$ из $\Delta$, противоречие.

Лемма 5. Если $\Gamma$ содержит $K_{4,6}$-подграф $\Delta=\left\{a_{1}, \ldots, a_{4} ; b_{1}, \ldots, b_{6}\right\}, K_{i}=$ $K_{i}(\Delta), x_{i}=\left|K_{i}\right|$, то $x_{3}=t=6$ и каждая точка $b_{i}$ смежна точно с тремя вершинами из $K_{3}$.

ДокАЗАтЕльство. Сначала заметим, что $x_{5}=0$. В противном случае для $d \in K_{5}$ некоторая точка $b_{j}$ не смежна $\mathrm{c} d, b_{j} \in K_{4}\left(\Delta-\left\{b_{j}\right\}\right), d \in K_{5}\left(\Delta-\left\{b_{j}\right\}\right)$, противоречие с тем, что $x_{0}\left(\Delta-\left\{b_{i}\right\}\right)+\sum\left(\begin{array}{c}i-1 \\ 2\end{array}\right) x_{i}\left(\Delta-\left\{b_{i}\right\}\right)=6$.

Если $K_{4}$ содержит точку $d$, то для любой не смежной с $d$ точки $b_{j}$ получим $x_{3}\left(\Delta-\left\{b_{j}\right\}\right)=0$. Пусть $b_{5}, b_{6} \notin[d], \Delta_{56}=\Delta-\left\{b_{5}, b_{6}\right\}$ и $z_{i}=x_{i}\left(\Delta_{56}\right)$. Тогда

$$
z_{0}+\sum\left(\begin{array}{c}
i-1 \\
2
\end{array}\right) z_{i}=9
$$

и $b_{5}, b_{6}, d \in K_{4}\left(\Delta_{56}\right)$. Отсюда $\left[b_{5}\right] \cap\left[b_{6}\right]$ содержит $t-3$ точки из $K_{3} \cup K_{4}$. Но $x_{0}+$ $\sum\left(\begin{array}{c}i-1 \\ 2\end{array}\right) x_{i}=t$, поэтому $\left[b_{5}\right] \cap\left[b_{6}\right]$ содержит $t-3$ точки из $K_{3}$. Пусть точка $b_{i}$ из $\left\{b_{1}, \ldots, b_{4}\right\}$ смежна не более, чем с $[(t-3) / 4]$ точками из $K_{3}$. Тогда $K_{3}\left(\Delta-\left\{b_{i}\right\}\right)$ содержит не менее $[3(t-3) / 4]$ точек из $K_{3}$ и $t-3 \leqslant 4$. Если $t=6$, то $b_{i}$ не смежна с точками из $K_{3}$ и $K_{3}\left(\Delta-\left\{b_{i}\right\}\right)$ содержит $t-3$ точек из [b5] $\left.\cap b_{6}\right]$. Кроме того, 
$d \in K_{3}\left(\Delta-\left\{b_{i}\right\}\right) \cup K_{4}\left(\Delta-\left\{b_{i}\right\}\right)$, противоречие. Значит, $x_{4}=0, x_{3}=t$ и имеется не более $3 t$ ребер между $K_{3}$ и $\left\{b_{1}, \ldots, b_{6}\right\}$.

Пусть $\left[b_{j}\right]$ содержит наименьшее число $\gamma$ точек из $K_{3}$. Тогда $\gamma \leqslant[t / 2]$. Для подграфа $\Delta_{j}=\Delta-\left\{b_{j}\right\}$ получим $b_{j} \in K_{4}\left(\Delta_{j}\right)$ и $K_{3}\left(\Delta_{j}\right)$ содержит $t-\gamma$ точек. Поэтому $t-\gamma \leqslant 3$ и $t=6$. Более того, $\gamma=3$ и каждая точка из $\left\{b_{1}, \ldots, b_{6}\right\}$ смежна точно с 3 точками из $K_{3}$. Лемма, а вместе с ней и теорема доказаны.

ДокАЗАТЕЛЬСТво СЛЕДСТВИЯ. Пусть точечный граф Г для обобщенного четырехугольника $G Q(4, t)$ содержит $K_{4,6}$-подграф. По лемме 5 получим $t=6$. Но тогда по утверждению (4) леммы 1.3 .4 [2] $\Delta$ содержится в $K_{4,7}$-подграфе из $\Gamma$. Противоречие с леммой 3.

Лемма 6. Пусть $\Delta=\left\{a_{1}, \ldots, a_{4} ; b_{1}, \ldots, b_{5}\right\}$ является $K_{4,5}$-подграфом точечного графа Г обобщенного четырехугольника порядка $(4, t), K_{i}=K_{i}(\Delta), x_{i}=\left|K_{i}\right|$. Eсли $t>8$, то либо $\Delta$ содержится в $(5,5)$-подграфе из $\Gamma$, либо $x_{3}=6$.

ДокАЗАТЕЛЬСтво. По лемме $4 x_{0}+\sum\left(\begin{array}{c}i-1 \\ 2\end{array}\right) x_{i}=6$, причем, если $w \in K_{0}$, то $t=4$. По условию каждое ребро подграфа $\Delta$ лежит на единственной прямой, причем $w$ смежна с единственной точкой на этой прямой. Но в этом случае множество $N$ из 20 точек указанного типа, смежных с $w$, должно совпадать с $[w]$. Противоречие с тем, что порядок максимальной клики из $N$ не больше 4 . Значит, $x_{0}=0$. Если $t>8$, то необходимо $t \geqslant 11$.

Пусть $x_{5}=0$. По следствию $K_{4}$ не содержит точек, смежных с 4 вершинами $a_{1}, \ldots, a_{4}$. Покажем, что $K_{4}$ не содержит точек, смежных с 4 вершинами из $\left\{b_{1}, \ldots, b_{5}\right\}$. Пусть вершина $w \in K_{4}$ смежна с вершинами $b_{1}, \ldots, b_{4}, \Delta^{\prime}=\left\{a_{1}, \ldots, a_{4} ; b_{1}, \ldots, b_{4}\right\}, L_{i}=K_{i}\left(\Delta^{\prime}\right)$. Тогда $\Delta^{\prime}$ является $(4,4)$-подграфом, $b_{5}, w \in L_{4}$, причем $\left[b_{5}\right] \cap[w]$ содержит $t-7$ точек из $L_{0}$, противоречие с тем, что $x_{0}\left(\Delta^{\prime}\right)+\sum\left(\begin{array}{c}i-1 \\ 2\end{array}\right) x_{i}\left(\Delta^{\prime}\right)=9$. Итак, $x_{4}=0$ и $x_{3}=6$.

ЛЕмма 7. Пусть $\Delta=\left\{a_{1}, \ldots, a_{3} ; b_{1}, \ldots, b_{n}\right\}$ является $(3, n)$-подграфом псевдогеометрического графа $Г$ для обобщенного четырехугольника порядка $(4,8), K_{i}=$ $K_{i}(\Delta), x_{i}=\left|K_{i}\right|$. Tогдa

$$
x_{0}+\sum\left(\begin{array}{c}
i-1 \\
2
\end{array}\right) x_{i}=3 n^{2}-28 n+81 \text {. }
$$

ДокАЗАТЕЛЬСТво. По условию $N=n+3, M=3 n, \sum\left(\begin{array}{c}d_{i} \\ 2\end{array}\right)=3 n(n+1) / 2$. По лемме 1 получим $x_{0}+\sum\left(\begin{array}{c}i-1 \\ 2\end{array}\right) x_{i}=3 n^{2}-28 n+81$.

ЛЕмма 8. Пусть псевдогеометрический граф Г для обобщенного четырехугольника порядка $(4, t)$ содерэит 4-клику $\Delta$, не лежсашую в 5-клике, $K_{i}=K_{i}(\Delta)$, $x_{i}=\left|K_{i}\right|$. Тогда

(1) $x_{0}+x_{3}=4 t+3$;

(2) если e $\in K_{3}, d \in \Delta-[e], \Delta^{\prime}=\Delta \cup\{e\}, L_{i}=K_{i}\left(\Delta^{\prime}\right), y_{i}=\left|L_{i}\right|$, то треугольник $\Delta \cap[e]$ изолирован в $[d] \cap[e], y_{0}=y_{0}+\sum_{i}\left(\begin{array}{c}i-1 \\ 2\end{array}\right) y_{i}=t+2, y_{2}=t+4 u$ если $t \geqslant 6$, то $L_{0}$ - коклика и каждая вершина из $L_{0}$ смежна $c t+1$ точкой uз $L_{2}$. 
ДокАЗАТЕЛЬСТВо. Подсчитав число ребер между $\Delta$ и $\Gamma-\Delta$ и число 2-путей с началом и конщом в $\Delta$ и средней вершиной вне $\Delta$, получим систему уравнений

$$
\sum_{i} x_{i}=20 t+1, \quad \sum_{i} i x_{i}=16 t+4, \quad x_{0}+\sum_{i}\left(\begin{array}{c}
i-1 \\
2
\end{array}\right) x_{i}=6 .
$$

Вычитая второе уравнение из суммы первого с третьим, получим первое утверждение леммы.

Пусть $x_{3} \neq 0, \Omega=\Gamma-\left(d^{\perp} \cup e^{\perp}\right)$. Тогда $|\Omega|=13 t-4$ и окрестности различных вершин треугольника $\Delta \cap[e]$ не пересекаются в $\Omega$. Покажем, что треугольник $\Delta \cap[e]=\{a, b, c\}$ изолирован в $[d] \cap[e]$. Пусть вершина $а$ смежна с некоторой вершиной $w \in([d] \cap[e])-\Delta)$. Тогда $[w]$ содержит не менее $4 t-3$ вершин из $\Omega,|[a] \cap \Omega|=4 t-1,[b]$ и $[c]$ содержат не менее, чем по $4 t-2$ вершин из $\Omega$. Итак, $|\Omega-([a] \cup[b] \cup[c])| \leqslant t+1$, a $[w] \cap \Omega$ содержит не более одной вершины из $[a]$ и не более $2 t-4$ вершин из $[b] \cup[c]$, следовательно, $2 t \leqslant t+1$, $t=1$, и $\mu=2$, противоречие с тем, что $\mu$-подграф $d^{\perp} \cap e^{\perp}$ содержит треугольник.

Теперь каждая вершина из $\{a, b, c\}$ смежна с $4 t-2$ вершинами из $\Omega$, поэтому $y_{0}=$ $y_{0}+\sum_{i}\left(\begin{array}{c}i-1 \\ 2\end{array}\right) y_{i}=t+2$. Далее, $L_{2}$ содержит $t-2$ вершин из $[d] \cap[e]$ и по одной вершине из $[d] \cap[z],[z] \cap[e]$ для $z \in\{a, b, c\}$, поэтому $y_{2}=t+4$. Если $f \in L_{0}$, то $|[f] \cap[z]|=t+1$ для $z \in \Delta^{\prime}$, следовательно, $[f]$ содержит не менее $t+1$ вершин из $L_{2}$.

Если $t \geqslant 6$, то $[f] \cap[g]$ содержит не менее $t-2$ вершин из $L_{2}$ для любых $f, g \in L_{0}$, поэтому $L_{0}$ является кокликой и $[f]$ содержит точно $t+1$ вершин из $L_{2}$ для любой вершины $f \in L_{0}$. Лемма доказана.

В леммах 9-11 предполагается, что вьполнены условия леммы 8 и $K_{3}$ содержит точку $e$, не смежную с точкой $d \in \Delta$. Зафиксируем обозначения

$$
\Lambda=\left\{L_{0} \cup\{d, e\} ; L_{2}\right\}, \quad \Lambda_{1}=L_{0} \cup\{d, e\}, \quad \Lambda_{2}=L_{2} .
$$

ЛЕмма 9. Параметр $t$ не больие 8.

ДокАЗАтЕЛьСтво. Допустим противное. Положим $\Omega=L_{2} \cap[d] \cap[e]$. Тогда $|\Omega|=$ $t-2$.

Для $w \in L_{2}$ число 2-путей с началом $w$, концом в $\Delta^{\prime}$ и средней вершиной в $\Gamma-\Delta^{\prime}$ равно $3 t+3$, если $w$ смежна с $d$ и $e$; равно $3 t+2$, если $w$ смежна с единственной вершиной из $\{d, e\}$. Если $[w]$ содержит $\gamma$ точек из $L_{2}$, то $[w]$ содержит $\gamma+t-1$ точек из $L_{0}$, если $w \in \Omega$, и $[w]$ содержит $\gamma+t$ точек из $L_{0}$, если $w \in L_{2}-\Omega$. Значит, $[u] \cap[w]$ содержит не менее $t-2$ точек из $L_{0}$ для любого ребра $u w$ из $L_{2}$. Для $t \geqslant 6$ подграф $L_{2}$ является кокликой, причем каждая точка из $\Omega$ смежна с $t-1$ вершиной из $L_{0}$, а каждая точка из $L_{2}-\Omega$ смежна с $t$ точками из $L_{0}$. Допустим, что $t>8$.

Пусть $f, g$ - такие две вершины из $L_{0}$, что $[f] \cap[g]$ содержит 5-элементное подмножество $\Omega_{0}$ из $\Omega$. Положим $\Phi=\left\{d, e, f, g ; \Omega_{0}\right\}, M_{i}=K_{i}(\Phi), z_{i}=x_{i}(\Phi)$. По лемме 4 $z_{0}+\sum\left(\begin{array}{c}i-1 \\ 2\end{array}\right) z_{i}=6$. Если $\Phi$ содержится в $(5,5)$-подграфе $\Psi$, то каждая вершина из $L_{0}-\Psi$ смежна со всеми точками из $L_{2}-\Omega_{0}$. Этот случай возникает лишь при $t \leqslant 6$. Значит, $z_{5}=0$. Каждая вершина из $L_{0}-\left(M_{3} \cup M_{4} \cup\{f, g\}\right)$ смежна со всеми точками из $L_{2}-\Omega_{0}$; а вершина из $L_{0} \cap M_{3}$ не смежна с единственной точкой из $L_{2}-\Omega_{0}$. В худшем случае (при $t=8$ и $z_{3}=6$ ) мы построим $K_{4,5}$-подграф с долями, лежащими в $L_{0}$ и $L_{2}-\Omega$, причем не менее 4 вершин из $M_{3}$ смежно с 4 точками этого $K_{4,5}$-подграфа. Противоречие с леммой 4.

Итак, $[f] \cap[g]$ содержит не более четырех вершин из $\Omega$ для любых различных $f, g \in L_{0}$. Отсюда $t \leqslant 8$. 
ЛЕмма 10. Если $t=8$, то двудольный подграф $\Lambda$ не содержит $K_{4,5}-u K_{3,6}$-подграфов.

ДокАЗАТЕЛЬСТво. Пусть $t=8$. Тогда $|\Lambda|=24$ и каждая вершина из доли $\Lambda_{i}$ не смежна точно с 3 точками из доли $\Lambda_{3-i}$. Допустим, что $\Lambda$ содержит двудольный подграф $\Sigma$. Положим $\Sigma_{i}=\Sigma \cap \Lambda_{i}, \Lambda_{i}^{\prime}=\Lambda_{i}-\Sigma_{i}$. Допустим, что $\Sigma$ является $K_{4,5}$-подграфом. Без ограничения общности $\left|\Sigma_{1}\right|=4$.

Число ребер между $\Sigma_{2}$ и $\Lambda_{1}^{\prime}$ равно 25 , и если $\Lambda_{1}^{\prime}$ содержит $\alpha_{i}$ точек из $K_{i}(\Sigma)$, то $\sum \alpha_{i}=8, \sum i \alpha_{i}=25$. Поэтому $\alpha_{4}=\alpha_{2}+1, \alpha_{3}=7-2 \alpha_{2}$. Противоречие с тем, что $\alpha_{3}+3 \alpha_{4} \leqslant 6$.

Допустим теперь, что $\Sigma$ является $K_{3,6}$-подграфом. Без ограничения общности, $\left|\Sigma_{1}\right|=3$. Число ребер между $\Sigma_{2}$ и $\Lambda_{1}^{\prime}$ равно 36 и по первому утверждению леммы $\Lambda_{1}^{\prime}$ не пересекает $K_{5}(\Sigma)$. Поэтому $\Lambda_{1}^{\prime}$ содержит 9 точек из $K_{4}(\Sigma)$. Противоречие с тем, что по лемме 7 верно равенство $x_{0}(\Sigma)+\sum\left(\begin{array}{c}i-1 \\ 2\end{array}\right) x_{i}(\Sigma)=21$.

Лемма 11. Если $t=8$, то $\Lambda$ не содержит $K_{3,5}$-подграфов.

ДокАЗАТЕЛЬСтво. Пусть $t=8, \Lambda$ содержит $K_{3,5}$-подграф $\Sigma$. Положим $\Sigma_{i}=\Sigma \cap \Lambda_{i}$, $\Lambda_{i}^{\prime}=\Lambda_{i}-\Sigma_{i}$. Без ограничения обшности, $\left|\Sigma_{1}\right|=3$.

Число ребер между $\Sigma_{2}$ и $\Lambda_{1}^{\prime}$ равно 30 , а между $\Sigma_{1}$ и $\Lambda_{2}^{\prime}-$ равно 12 . Покажем сначала, что $K_{0}(\Sigma)$ не пересекает $\Lambda_{2}^{\prime}$. В противном случае $\Lambda_{2}^{\prime}$ содержит точку $w$ из $K_{0}(\Sigma)$ и 6 точек $f_{1}, \ldots, f_{6}$ из $K_{2}(\Sigma)$. Далее, $\Lambda_{1}^{\prime} \subset[w]$ и $\left[f_{i}\right]$ содержит 7 точек из $\Lambda_{1}^{\prime}$. Из леммы 10 следует, что для любых различных $i, j \in\{1, \ldots, 6\}$ подграф $\left[f_{i}\right] \cap\left[f_{j}\right]$ содержит точно 5 точек из $\Lambda_{1}^{\prime}$ и $\Lambda_{1}^{\prime} \subset\left[f_{i}\right] \cup\left[f_{j}\right]$. Таким образом, пары точек из $\Lambda_{1}^{\prime}$, не смежные с $f_{i}$, должны попарно не пересекаться. Противоречие с тем, что $\left|\Lambda_{1}^{\prime}\right|=9$.

Итак, $K_{0}(\Sigma)$ не пересекает $\Lambda_{2}^{\prime}$ и $\Lambda_{2}^{\prime}$ содержит 2 точки $w_{1}, w_{2}$ из $K_{1}(\Sigma)$ и 5 точек $f_{1}, \ldots, f_{5}$ из $K_{2}(\Sigma)$. Пусть $a$ - точка из $\Sigma_{1}$, не смежная с $w_{1}$ и $w_{2}$. Тогда $a$ не смежна с некоторой точкой $f_{i}$ и мы получим $K_{3,5}$-подграф $\Sigma^{\prime}=\left\{w_{1}, w_{2}, f_{i} ;\left[w_{1}\right] \cap\left[w_{2}\right] \cap\left[f_{i}\right] \cap \Lambda_{1}\right\}$, причем $a \in K_{0}\left(\Sigma^{\prime}\right)$, противоречие. Лемма доказана.

ДоКАЗАТЕЛЬСТВо ПРЕДЛОЖЕНИЯ 3. Можно считать, что $\mu$-подграф $[d] \cap[e]$ содержит треугольник, поэтому вьполняются леммы 8-11. Число ребер между $L_{2}-([d] \cap[e])$ и $L_{0}$ равно 48 , поэтому некоторая точка $f$ из $L_{0}$ смежна не более, чем с 4 вершинами из $L_{2}-([d] \cap[e])$ и мы получим $K_{3,5}$-подграф $\{d, e, f ;[d] \cap[e] \cap[f]\}$. Противоречие с леммой 11. Предложение 3 доказано.

\section{СПИСОК ЦИТИРОВАННОЙ ЛИТЕРАТУРЫ}

[1] Браувер А.Е., ван Линт Й.Х. Сильно регулярные граффы и частичные геометрии // Кибернетический сборник. 1987. Т. 24. С. 186-229.

[2] Paine S., Thas J. Finite generalized quadrangles. Boston: Pitman, 1985.

[3] Haemers W. There exists no $(76,21,2,7)$ strongly regular graph // Finite Geometries and Combinatorics, London Math. Soc. Lecture Notes / ed. F. De Clerck et. al. V. 191. Cambridge: Cambridge Univ. Press, 1993. P. 175-176.

[4] Махнев А. А. О псевдогеометрических граффах некоторых частичных геометрий // Вопросы алгебры. Вып. 11. Гомель: Изд-во Гомельского университета, 1997. С. 60-67.

[5] Wilbrink H. A., Brouwer A. E. (57, 14,1) strongly regular graph does not exist // Proc. Kon. Nederl. Akad. Ser. A. 1983. V. 45. № 1. P. 117-121. 